\section{PTU-259 STENT PLACEMENT IN PALLIATION OF OESOPHAGO- GASTRIC CANCER: THE STORY SO FAR}

doi:10.1136/gutjnl-2012-302514c.259

'S F Neong, * 2J Bhattacharyya, ${ }^{3} \mathrm{~A}$ Leahy, ${ }^{2}$ I R Sargeant, ${ }^{4} \mathrm{D}$ L Morris. ${ }^{1}$ Department of Gastroenterology, Charing Cross Hospital, London, UK; ${ }^{2}$ Department of Gastroenterology, Lister Hospital, London, UK; ${ }^{3}$ Department of Gastroenterology, Watford General Hospital, Hertfordshire, UK; ${ }^{4}$ Department of Gastroenterology, OE II Hospital, Hertfordshire, UK

Introduction The majority of patients with oesophago-gastric cancer are diagnosed at an advanced incurable stage where palliation of symptoms remain the cornerstone of management. Most centres now palliate patients endoscopically with stent insertion, argon plasma coagulation and laser therapy.

Methods This is a retrospective review of the overall use of palliative stenting in patients with advanced oesophageal and gastric cancer across 2 acute hospital trusts in Hertfordshire with a catchment population of approximately 1 million in the 1-year period between 1 April 2010 and 31 March 2011. We looked through the endoscopy reports, medical notes and our prospectively maintained Upper Gastrointestinal Cancer database for any reported post procedural complications and calculated the 7,14 and 30-day mortality rate for this cohort of patients.

Results 30 patients in West Hertfordshire NHS Trust (WHHT) and 19 patients in East \& North Hertfordshire NHS Trust (E\&NH) had stent insertion where the median age is 76 years. $53 \%$ of patients in E\&NH were diagnosed with squamous cell carcinoma compared to $20 \%$ in WHHT. $53 \%$ of all patients stented in WHHT received both intravenous sedation and pharyngeal anaesthesia in comparison to E\&NH where $21 \%$ had the same combination. The main type of stent deployed is the Boston Scientific Ultraflex covered metal stent, with only $3 \%$ of patients receiving an uncovered metal stent. All the stents were deployed successfully. Within 3 months of insertion, stent migration was reported in six patients (12\%), all of whom had covered metal stents. None of our patients had perforation or haemorrhage post procedure. Our 14 and 30-day mortality is 7\% and $18 \%$ respectively which mirrors, if not lower than the national figures of $8 \%$ and $19 \%$.

Conclusion The difference in histology between the two trusts reflects additional use of laser therapy in E\&NH. The reliability of reporting of complications to the national audit was questioned at the recent British Society of Gastroenterology Meeting 2011. The apparent increase in our complication rates is likely to reflect the more accurate reporting across the region due to the existence of a robust prospectively maintained database. Our patient selection seemed appropriate given that most of them survive for more than 30 days. Uncovered stent or laser therapy should be considered in appropriate patients to reduce stent migration rates. The effectiveness of the procedure can be evaluated with a standardised dysphagia scoring system. Our audit is leading towards a change in stent management across the network.

Competing interests None declared.

\section{PTU-260 ARE WE WORKING AT A LOSS? DOES ROUTINE CODING FOR ENDOSCOPIC RETROGRADE CHOLANGIOPANCREATOGRAPHY (ERCP) PROVIDE ACCURATE REMUNERATION?}

doi:10.1136/gutjnl-2012-302514c.260

${ }^{1} \mathrm{~V}$ Jackson, ${ }^{*}{ }^{2} \mathrm{~L}$ Siadankay, ${ }^{3} \mathrm{~B}$ Saleh, ${ }^{4} \mathrm{R}$ Sturgess, ${ }^{4} \mathrm{~N}$ Stern. ${ }^{1}$ Endoscopy, Aintree University Hospital NHS Foundation Trust, Liverpool, UK; ${ }^{2}$ Clinical Audit, Aintree University Hospital NHS Foundation Trust, Liverpool, UK; ${ }^{3}$ Finance, Aintree University
Hospital NHS Foundation Trust, Liverpool, UK; ${ }^{4}$ Department of Gastroenterology, Aintree University Hospital NHS Foundation Trust, Liverpool, UK

Introduction Endoscopic procedures in the UK are remunerated through "Payment by results" with a healthcare resource group (HRG) tariff assigned to each procedure. HRG tariffs depend on accurate coding of the diagnosis and procedure to enable appropriate payment from primary care trusts (PCT). There is little existing data about the accuracy of coding in ERCP to generate appropriate HRG tariffs.

Methods Cases were randomly selected from those attending for ERCP at our unit between 24 March 2010 and 2 July 2010 with full information available. Each procedure was reviewed by a member of the hospitals coding department and a consultant gastroenterologist who regularly performs ERCP, with details of initial routine coding available. Cases were all re-coded following clinician input with subsequent re-calculation of HRG tariff.

Results 39 cases were reviewed. 20 in-patient procedures and 19-day cases. This included 24 (61.5\%) accurately coded and $15(38.5 \%$, 2 -day cases and 7 in-patients) requiring re-coding. The re-coding was due to incorrect primary diagnostic code: 3 ; primary procedure code: 9; both codes: 3 . Four of the procedure code changes resulted in increases to HRG tariffs. None of the re-coding led to a reduction in tariff. Three of these were due to incorrect coding of metal stent insertion and one due to omission of coding for sphincterotomy. The initial coding led to an income of $£ 60033$ with the revised coding suggesting remuneration should have been $£ 69037$ : a shortfall of $£ 9004$. The single biggest shortfall was in the coding of "stent" insertion. Unless clearly labelled as "metal stent," these were routinely coded as plastic stents which carry a lower tariff.

Conclusion There is a difference in coding of ERCPs in $38.5 \%$ of procedures we studied. This led to over $10 \%$ re-coded to a higher HRG tariff, potentially increasing ERCP related income. Accurate coding is necessary to ensure appropriate remuneration for hospital trusts. Particular attention to the recording of insertion and coding of metallic stents is needed.

Competing interests None declared.

\section{REFERENCE}

1. Moar K, Rogers S. Impact of coding errors on departmental income: an audit of coding of microvascular free tissue transfer cases using OPCS-4 in UK. Br J Oral Maxillofac Surg 2012;50:85-7.

\section{PTU-261 ALCOHOL MISUSE: REFER AND DETER. REDUCING RE-ADMISSION RATES FOR INPATIENT DETOXIFICATION}

doi:10.1136/gutjnl-2012-302514c.261

V L Beckett,* J Ching, V Wood, G Singh. Department of Gastroenterology, Bassetlaw District General Hospital, Worksop, UK

Introduction Alcohol abuse and related diseases are growing public health concerns. In the UK $26 \%$ of adults consume harmful quantities of alcohol. ${ }^{1}$ Alcohol related admissions are increasing by $11 \%$ each year. ${ }^{2}$ Yorkshire and Humber encompass one of the highest rates of alcohol misuse in the UK. NICE have developed a National Alcohol Strategy to target these issues which cost the UK economy $£ 23$ billion and NHS $>£ 2.7$ billion per year. ${ }^{3}$ Despite initiatives to improve the quality of care and improve continuity between primary and secondary care, $58 \%$ of UK acute medical units lack an alcohol support service.

Methods We retrospectively audited the impact of the Alcohol specialist nurse (ASN) on readmission for inpatient detoxification between 2004 and 2011 at our hospital. This followed the establishment of an Alcohol Care Team in 2005 as recommended by The British Society of Gastroenterology. ${ }^{2}$ Medical and surgical inpatients 